\title{
MONOCAPAS AUTO-ENSAMBLADAS DE ALQUENOS EN SUPERFICIES HIDROGENADAS DE SILICIO (100)
}

\author{
Yris Del Pilar Obregón Rodríguez ${ }^{* a}$, César R. Mayor Sánchez
}

\begin{abstract}
RESUMEN
La preparación en la formación de monocapas auto-ensambladas en las superficies de silicio nos ha permitido el avance tecnológico de nuevos materiales semiconductores orgánicos, obteniéndose dispositivos que sobresalen por sus propiedades eléctricas a escala nanométrica ${ }^{1}$. Para esta investigación, se consideró la reacción entre alquenos y la superficie hidrogenada de Si (100) para formar monocapas orgánicas auto-ensambladas ${ }^{2}$. Las moléculas orgánicas de alquenos que se utilizaron fueron 1-dodeceno, 1-tetradeceno, 1-hexadeceno y 1-octadeceno, los cuales resaltan para el proceso de funcionalización de estas películas orgánicas. Para el tratamiento de la superficie de silicio se tomó en cuenta una previa reacción de hidrogenación ${ }^{3,4}$, obteniéndose grupos funcionales $\mathrm{Si}-\mathrm{H}$ en la superficie. Respecto a la reacción de formación de la monocapa orgánica, el sustrato de silicio de superficie hidrogenada reaccionó con cada uno de los alquenos ${ }^{5}$ mencionados a 220 oC por 2 horas. Las técnicas experimentales usadas como la técnica de AFM (Atomic Force Microscopy), la medición del ángulo de contacto entre gotas de agua y el sustrato, y el step potential nos verifican la presencia de la superficie hidrogenada y las monocapas orgánicas auto-ensambladas en la superficie.
\end{abstract}

Palabras clave: Monocapa auto-ensamblada, superficie hidrogenada, alquenos, microscopía de fuerza atómica.

\section{SELF-ASSEMBLED MONOLAYERS OF ALKENES ON HYDROGEN-TERMINATED SILICON (100) SURFACES}

\begin{abstract}
The preparation in the formation of self-assembled monolayers on silicon surfaces has allowed us the technological advancement of new organic semiconductor materials, obtaining devices that stand out for their electrical properties at the nanometric scale ${ }^{1}$.

For this investigation, the reaction between alkenes and hydrogenated surface of Si (100) was considered to form self-assembled organic monolayers ${ }^{2}$. The organic molecules of alkenes used were 1-dodecene, 1-tetradecene, 1-hexadecene and 1-octadecene, which stand

\footnotetext{
a Facultad de Ciencias e Ingeniería - Sección Química, Pontificia Universidad Católica del Perú (PUCP), Av. Universitaria 1801, San Miguel, Lima 32, Perú, yares2605@gmail.com

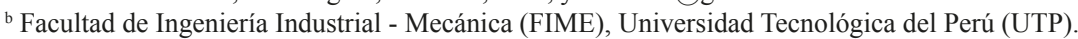


out for the functionalization process of these organic films. For the treatment of the silicon surface, a previous hydrogenation ${ }^{3,4}$ reaction was considered, obtaining $\mathrm{Si}-\mathrm{H}$ functional groups on the surface. Regarding, the formation reaction of the organic monolayer, the silicon substrate of the hydrogenated surface reacted with each of the alkenes ${ }^{5}$ at $220^{\circ} \mathrm{C}$ for 2 hours. The experimental techniques used as the AFM (Atomic Force Microscopy) technique, the measurement of the contact angle between water droplets and the substrate, and the potential step verify the presence of the hydrogenated surface and the self-assembled organic monolayers on the surface.

Key words: Self-assembled monolayer, hydrogen-terminated surface, alkenes, atomic force microscopy.

\section{INTRODUCCIÓN}

Este trabajo de investigación realza el gran interés por el estudio en la preparación de monocapas orgánicas auto-ensambladas sobre silicio con fines aplicativos en sistemas electroquímicos de reconocimiento molecular y tecnológico, ya que en estos últimos años han sido ampliamente investigadas y desarrolladas ${ }^{2,6}$.

A partir del año 1970, la fabricación de sensores químicos con tecnología microelectrónica ha sido una mejor alternativa frente a los clásicos métodos de detección de laboratorio. El crecimiento tecnológico logró la integración de dos tecnologías: la tecnología microelectrónica para la fabricación de circuitos integrados y la tecnología de materiales selectivos a iones para la fabricación de ISE's (Ion-selective electrodes), teniendo como única herramienta de estudio el silicio en el área de la microelectrónica ${ }^{3,4,7}$.

El silicio como componente de la electrónica y computadoras, unidos literalmente a materiales orgánicos y biológicos crearía un nexo entre el mundo electrónico y el mundo biológico en sus inicios ${ }^{2,8,9}$.

A principios de la segunda mitad del siglo pasado se empezó la investigación en la electrónica molecular logrando una gran explotación por su potencial tecnológico en el estudio de los materiales semiconductores orgánicos en dispositivos electrónicos ${ }^{3}$ de gran competencia en comparación a las prestaciones de algunos dispositivos tradicionales con semiconductores inorgánicos ${ }^{6}$.

Hoy, la llamada electrónica orgánica se enfoca en esta clase de estructuras moleculares desarrolladas tanto en el campo tecnológico como en el científico, basándose en la alteración de las propiedades eléctricas de nuevos materiales semiconductores orgánicos a escala nanométrica y la miniaturización de dispositivos electrónicos de nueva generación ${ }^{1}$.

Para, finalmente, diseñar sustratos modificados con monocapas auto-ensambladas que ofrecen diversas alternativas de preparar dispositivos particulares para el estudio de distintos 
procesos fisicoquímicos, como destacan los fenómenos de transferencia electrónica a distancias controladas de la interfase ${ }^{2,9}$.

\section{PARTE EXPERIMENTAL}

\section{Tratamiento preliminar de la superficie de silicio}

Se colocó una pieza de silicio de aproximadamente $1 \times 1 \mathrm{~cm}^{2}$ en una solución de $\mathrm{H}_{2} \mathrm{O}_{2}$ al $20 \%$ y $\mathrm{NH}_{4} \mathrm{OH}(5: 1)$, a $80^{\circ} \mathrm{C}$, con agitación constante durante 30 minutos. Luego, la pieza de silicio fue enjuagada con abundante agua destilada. Nuevamente, la pieza de silicio fue llevada a una solución del $10 \%$ de HF por 45 segundos para luego ser enjuagada con abundante agua destilada. Por último, la pieza fue llevada a una solución de $\mathrm{H}_{2} \mathrm{O}_{2}$ al $30 \%$ y $\mathrm{H}_{2} \mathrm{SO}_{4}(1: 2)$ por 2 horas a $80^{\circ} \mathrm{C}$ para luego ser nuevamente enjuagada con abundante agua destilada por 5 minutos ${ }^{5}$.

\section{Preparación de monocapas con alquenos}

La preparación de las monocapas se realizó con alquenos (dodeceno, $\mathrm{C}_{12}$; tetradeceno, $\mathrm{C}_{14}$; hexadeceno, $\mathrm{C}_{16}$ y octadeceno, $\mathrm{C}_{18}$ ). Para esto se procedió a la pasivación de la pieza de silicio usando una solución de $\mathrm{HF}$ al $2 \%$ por un tiempo de 2 minutos.

Luego, la pieza de silicio fue llevada a un tubo de ensayo con $20 \mathrm{ml}$ de 1-alqueno $\left(\mathrm{C}_{12}, \mathrm{C}_{14}\right.$, $\mathrm{C}_{16} \mathrm{y}_{18}$ ), previamente desoxigenada, haciendo burbujear nitrógeno por espacio de 1 hora, $\mathrm{y}$ se colocó el tubo de ensayo en un baño de aceite entre $200^{\circ} \mathrm{C}-220^{\circ} \mathrm{C}$ por 2 horas haciendo burbujear nitrógeno constantemente para evitar la presencia de oxígeno durante la formación de la monocapa ${ }^{2,5,9}$. Finalmente, la pieza de silicio es secada con nitrógeno.

\section{Técnicas de caracterización de monocapas con alquenos}

Microscopía de Fuerza Atómica (AFM). Para cerciorarnos de la formación de monocapas orgánicas en el sustrato de silicio, se realizó en primera instancia la verificación de la elección de un buen tratamiento de pasivación en las muestras de superficie de silicio con el registro de imágenes topográficas por AFM para los casos de muestras de $\mathrm{SiO}_{2}$ (óxido de silicio) y superficie de silicio hidrogenadas $(\mathrm{Si}-\mathrm{H})$ libre de óxidos, es decir, antes y después de la pasivación. Esta evaluación se realizó de manera cualitativa ${ }^{3,8}$.

Las imágenes fueron obtenidas con el equipo EasyScan 2 del fabricante Nanosurf®, en modo de contacto con resolución de 256 x 256 píxel, sistema de piezo barrido de 5 x $5 \mathrm{~mm}^{2}$ y sonda tipo ultralever de $600 \mathrm{~nm}$ de diámetro. Estos ensayos fueron realizados en el Laboratorio de Microscopía de Fuerza Atómica del Instituto Peruano de Energía Nuclear (IPEN), siendo las mejores imágenes obtenidas usando el equipo mencionado.

Ángulo de contacto. La medición del ángulo de contacto es otra de las técnicas para determinar la presencia de monocapas sobre sustratos sólidos que consiste en analizar las características hidrofóbicas o hidrofílicas de las mismas ${ }^{5}$. 
Para este ensayo se empleó $0,3 \mathrm{ml}$ de agua destilada sobre la superficie con la monocapa orgánica auto-ensamblada, del cual se midió el ángulo de contacto respecto al sustrato. De tales mediciones del ángulo de contacto se obtuvieron imágenes fotografiadas de las gotas que se realizaron tanto en el Laboratorio 2 de Química (PUCP) como en el Laboratorio de Materiales Nanoestructurados (UNI).

Respuesta electroquímica (step potential). Para estas mediciones de respuesta electroquímica, se logró montar las muestras de silicio en una celda de tres electrodos y se les aplicó potenciales del tipo escalón ${ }^{6,10}$. Para esta evaluación se realizó antes la aplicación del voltaje escalón, en la cual se mantuvo el voltaje de circuito abierto (VOC) por 10 segundos (para un primer tiempo). Luego se aplicó un voltaje escalón de $-0,5 \mathrm{~V}$ de amplitud por un intervalo de 10 segundos (para un segundo tiempo), al cabo de los cuales, el sistema regresa a la condición de voltaje VOC, generando curvas de corriente por los sustratos de silicio con diferentes cadenas carbonadas de alquenos (monocapas orgánicas auto-ensambladas). Para este estudio se contó también con la colaboración del Laboratorio de Materiales Nanoestructurados (UNI).

\section{RESULTADOS Y DISCUSIÓN}

\section{Microscopía de fuerza atómica (AFM)}

El análisis cualitativo de la imagen de la figura 1 nos detalla un sustrato uniforme y parcialmente liso de la misma tonalidad en la totalidad de la superficie.

En esta imagen se presume la presencia del $\mathrm{SiO}_{2}$ (óxido de silicio) adherida a la superficie, sin ningún tipo tratamiento de pasivación.

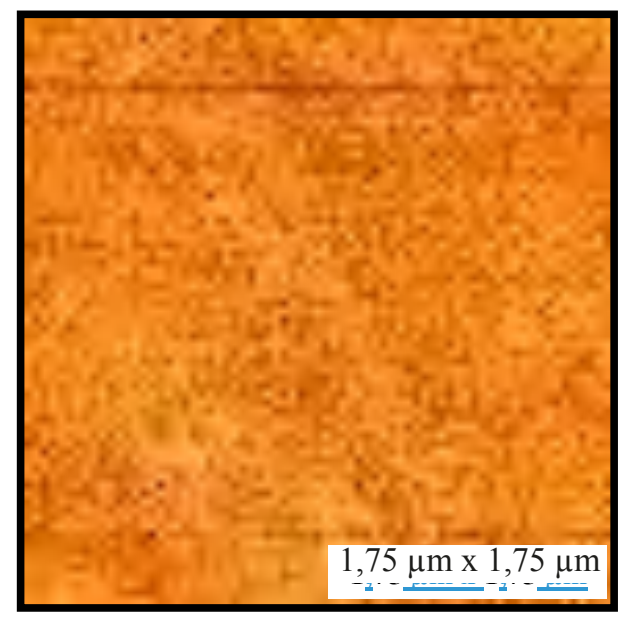

Figura 1. Superficie no tratada $\left(\mathrm{SiO}_{2}\right)$. 
El análisis cualitativo de la imagen de la figura 2 nos muestra una superficie densamente granulada, con mayor rugosidad y de tonalidad más clara que la imagen anterior.

Se puede predecir la adhesión del hidrógeno en la superficie de silicio ( $\mathrm{Si}-\mathrm{H})$ libre de óxidos presentes en el sustrato debido al tratamiento de pasivación

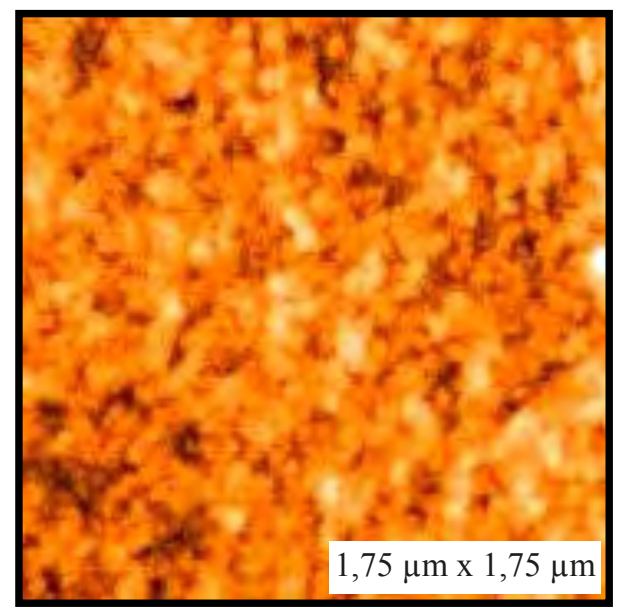

Figura 2. Superficie hidrogenada Si-H (libre de óxido).

\section{Ángulo de contacto}

A continuación, se mostrarán las micrografías de las imágenes tomadas y analizadas en este estudio:

La figura 3 muestra la microfotografía de las gotas de agua destilada sobre el sustrato de silicio sin tratamiento (se presume la presencia del óxido de silicio natural, $\mathrm{SiO}_{2}$ ), con un ángulo de $48,3^{\circ}$; cuyo ángulo es tomado de la tangente del borde del lado derecho con respecto a la base de la superficie de silicio.

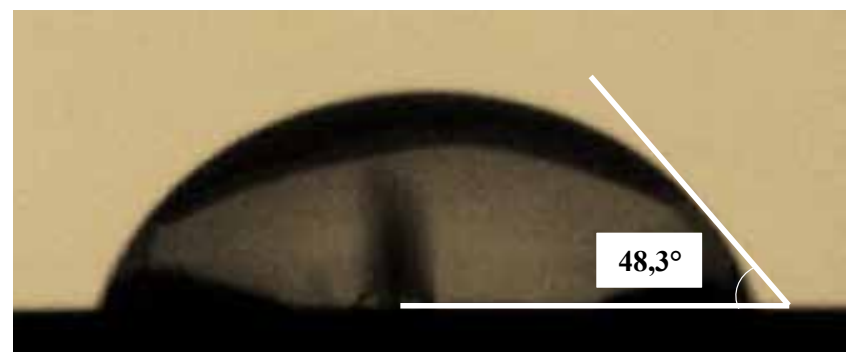

Figura 3. Ángulo de contacto de una superficie no pasivada $\left(\mathrm{SiO}_{2}\right)$. 
La microfotografía de la figura 4 muestra la gota de agua destilada sobre la superficie de silicio hidrogenado $(\mathrm{Si}-\mathrm{H})$. Se observa claramente que la superficie de silicio pasivada es altamente hidrofílica con un ángulo de contacto de $11,0^{\circ}$ (medido del lado derecho), esto debido a la presencia de las fuerzas intermoleculares entre adsorbato y el sustrato.

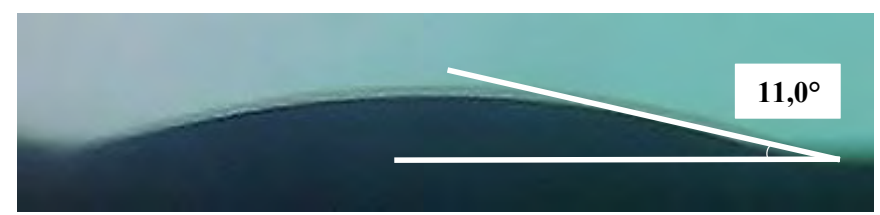

Figura 4. Ángulo de contacto de una superficie hidrogenada (Si-H).

La microfotografía presentada en la figura 5 muestra la alta hidrofobicidad presente entre el adsorbato (agua destilada) y el sustrato (monocapa auto-ensamblada del alqueno 1-dodeceno), con un ángulo de contacto de $87,6^{\circ}$.

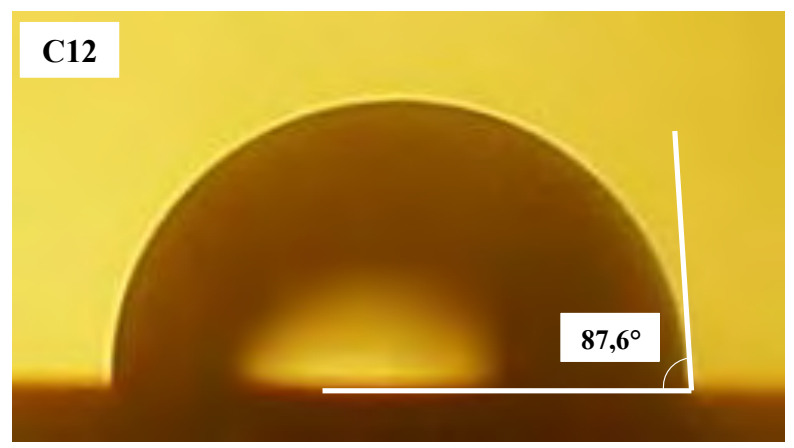

Figura 5. Ángulo de contacto de una monocapa orgánica sobre silicio (silicio dodecano).

La microfotografía de la figura 6 muestra claramente la hidrofobicidad presente entre el adsorbato (agua destilada) y el sustrato (monocapa auto-ensamblada del alqueno 1-tetradeceno), con un ángulo de contacto de $93,6^{\circ}$.

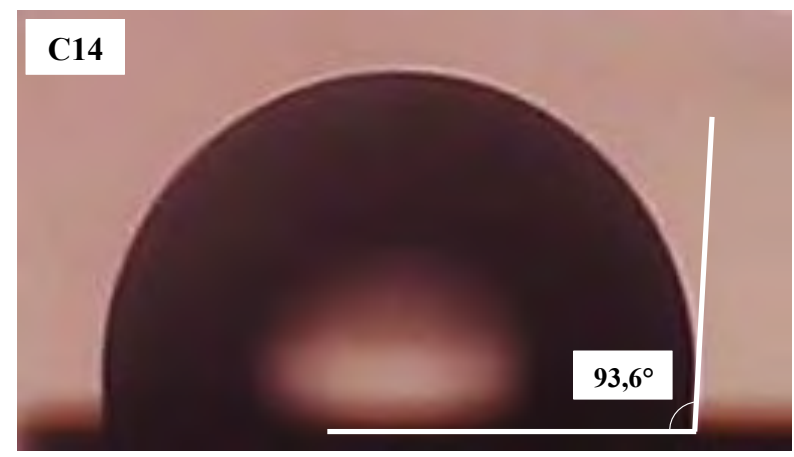

Figura 6. Ángulo de contacto de una monocapa orgánica sobre silicio (silicio tetradecano). 
La microfotografía presentada en la figura 7 señala la alta hidrofobicidad con un ángulo de contacto de $87,6^{\circ}$ entre el adsorbato (agua destilada) y el sustrato (monocapa auto-ensamblada del alqueno 1-hexadeceno).

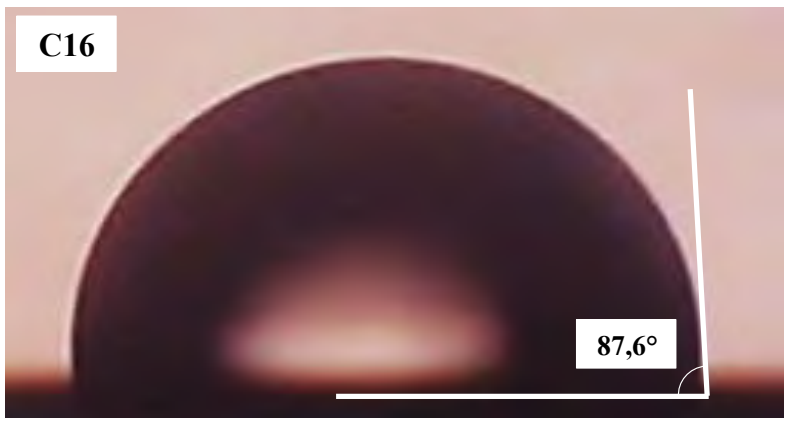

Figura 7. Ángulo de contacto de una monocapa orgánica sobre silicio (silicio hexadecano).

La microfotografía de la figura 8 señala claramente la hidrofobicidad con un ángulo de contacto de $87,4^{\circ}$ entre el adsorbato (agua destilada) y el sustrato (monocapa auto-ensamblada del alqueno 1-octadeceno).

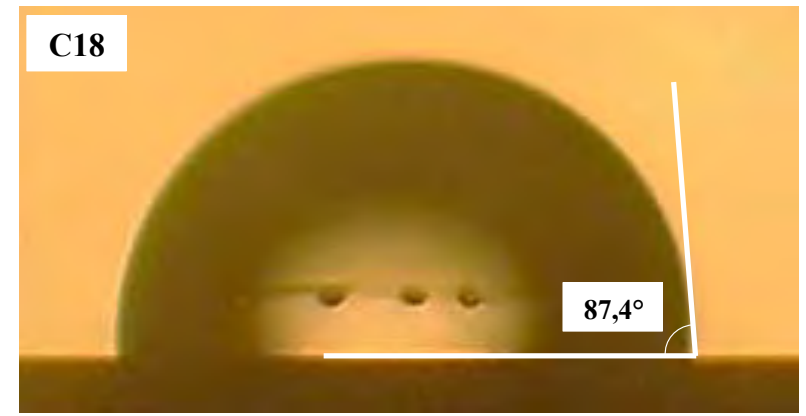

Figura 8. Ángulo de contacto de una monocapa orgánica sobre silicio (silicio octadecano).

Las micrografías que corresponden a los sustratos de silicio con alquenos (monocapas orgánicas auto-ensambladas) muestran un comportamiento hidrofóbico, lo que indica alta hidrofobicidad (entre la cadena alquílica auto-ensamblada sobre la superficie de silicio y el agua), posiblemente debido a la ausencia de interacciones por puente de hidrógeno, presentando ángulos de contacto entre $87,4^{\circ}-93,6^{\circ}$.

Es importante notar que no se puede establecer una diferencia sistemática entre ángulo de contacto y número de carbonos de los alquenos, probablemente estas diferencias están dentro del límite de incertidumbre del instrumento. 


\section{Respuesta electroquímica (step potential)}

Las curvas de corriente que se generaron por la medición de sustratos de silicio con diferentes cadenas carbonadas de alquenos (monocapas orgánicas auto-ensambladas) se pueden visualizar en la figura 9.

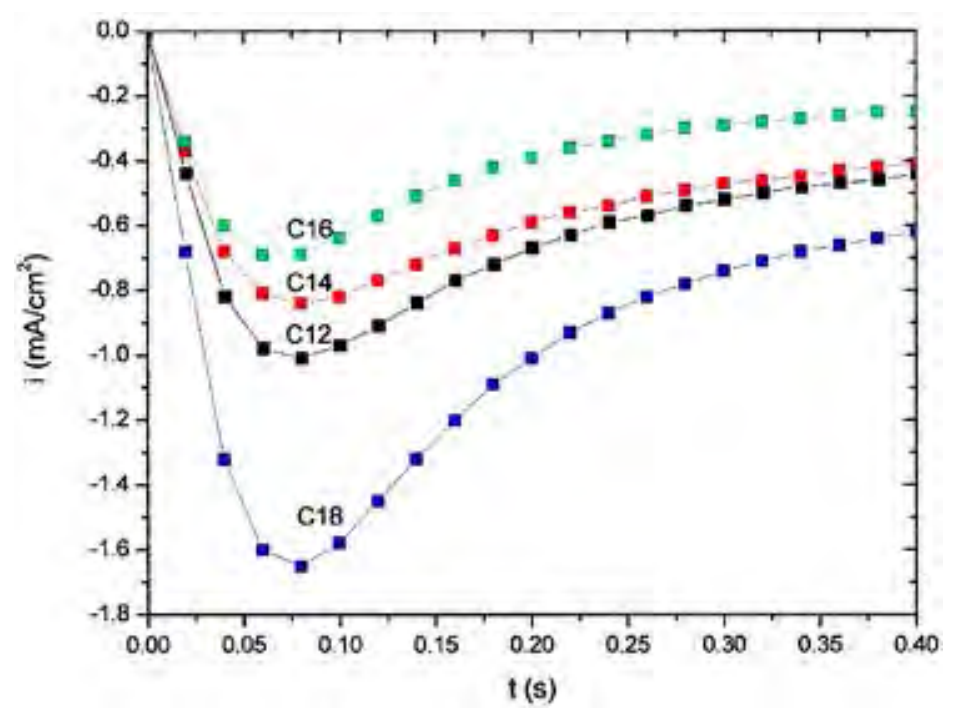

Figura 9. Respuesta de corriente ante el voltaje escalón para sustratos con monocapas de diferentes alquenos.

De los resultados obtenidos experimentalmente, se puede observar que en los diagramas de corriente existe una evidente disminución de flujo de corriente en valor absoluto conforme aumenta el número de carbonos en la cadena desde $\mathrm{C}_{12}-\mathrm{C}_{16}$ (figura 9), verificando un buen empaquetamiento de las moléculas orgánicas en el sustrato.

Sin embargo, esta tendencia se rompe en el caso del 1-octadeceno, cuyo valor mínimo debió ser menor que el del 1-hexadeceno. Una de las razones para lo anterior sería las imperfecciones físicas del sustrato que son un impedimento para que el adsorbato se adhiera en la superficie como también su baja densidad de empaquetamiento en el sustrato debido al mayor número de carbonos en la cadena.

En esta investigación también se consideró, inicialmente, la posibilidad de caracterizar las monocapas auto-ensambladas de alquenos usando la técnica de Espectrometría Infrarroja por Transformadas de Fourier (FTIR).

En las pruebas preliminares de esta investigación se hicieron lecturas en IR (con el equipo Perkin Elemer FTIR - 1600, usando un cristal de Si para ATR) con otros compuestos orgánicos como bromuros alquílicos y compuestos aromáticos como fenol y anilina, en superficies de silicio hidrogenada, generando resultados con un éxito parcial (no contundente). 
Como se sabe, la técnica de FTIR es una herramienta útil para la investigación de especies funcionalizadas y no funcionalizadas de una superficie químicamente modificada. Asimismo, esta herramienta de trabajo cuenta con acoplamientos como también de ciertas condiciones particulares $^{11-13}$. Sin embargo, los ensayos experimentales preliminares que realizamos mostraron baja intensidad en las regiones de estiramiento (como $\mathrm{C}-\mathrm{H}$ ) y no mostraron una clara presencia de los grupos funcionales en la superficie de silicio.

Quizás la poca estabilidad de la monocapa y/o la exposición a la atmósfera, a la cual está sometida el sustrato para su lectura, causó esta variante en la región espectral como se registra también en los artículos reportados por el grupo de Linford $^{4}$, Sieval ${ }^{14}$ y por la investigadora Meng $\mathrm{Li}^{13}$.

Cabe resaltar que los resultados obtenidos por la técnica de FTIR en otras investigaciones de referencia ${ }^{11,12,15}$, sí han sido de utilidad para este tipo de investigación; sin embargo, hay que mencionar que los equipos que usaron esos grupos de investigación han sido muy sofisticados, obteniendo ellos resultados útiles en la caracterización de las monocapas autoensambladas en los sustratos.

\section{CONCLUSIONES}

- Se verifica que el buen tratamiento de pasivación que se aplica a los sustratos de óxido de silicio natural nos resulta una superficie de silicio libre de óxidos, es decir, una superficie de silicio apta para ser unida covalentemente al hidrógeno (formación de Si-H).

- Se ha comprobado por las técnicas del ángulo de contacto y step potential, la presencia de monocapas alquílicas con una activación térmica menor a $220^{\circ} \mathrm{C}$ y en condiciones de protección atmosférica menos rigurosas que las reportadas por Sieval y colaboradores ${ }^{14}$.

- Los resultados de las imágenes topográficas por AFM muestran que las superficies pasivadas con hidruros (Si-H), libres de óxidos están preparadas para el autoensamblaje con las moléculas orgánicas.

- Se ha verificado la presencia de la monocapa orgánica en la superficie de silicio bajo la técnica del step potential (comportamiento electroquímico) y la técnica del ángulo de contacto (comportamiento hidrofóbico).

\section{AGRADECIMIENTO}

Los autores agradecen el apoyo brindado para este trabajo de investigación al doctor Abel Gutarra del Laboratorio de Materiales Nanoestructurados de la Facultad de Ciencias (UNI), como también, al doctor José Solís del Laboratorio de Microscopía de Fuerza Atómica Instituto Peruano de Energía Nuclear (IPEN). 


\section{REFERENCIAS BIBLIOGRÁFICAS}

1. M. Más Montoya, "Síntesis de Nuevos Sistemas Heteroacénicos y Estudio de sus Propiedades como Semiconductores Orgánicos para su Aplicación en Electrónica Molecular", tesis doctoral para optar el título de Doctor en Ciencias Químicas, Universidad de Murcia, 2015.

2. A.B. Sieval, R. Linke, G. Heij, G. Meijer, H. Zuilhof, E. J. R. Sudhölter, Langmuir, 2001, 17(24), 7554.

3. T. Ishizaki, N. Saito, L. Sunhyung, K. Ishida, T. Osamu, Langmuir, 2006, 22(24), $9962-$ 9966.

4. M.R. Linford, P. Fenter, P. M. Eisenberger, Ch. E. D. Chidsey, J. Am. Chem. Soc., 1995, $117(11), 3145-3155$.

5. A.B. Sieval, V. Vleeming, H. Zuilhof, E. J. R. Sudhölter, Langmuir, 1999, 15(23), 82888291.

6. D.A. Gamero, "Desarrollo de electrodos modificados con matrices de sílice para posibles aplicaciones en sensores y biosensores electroquímicos", tesis doctoral para optar el título de Doctor en Ciencias Químicas, Universidad Alicante, 2014.

7. P. Thissen, O. Seitz, Y.J. Chabal, Progress in Surface Science, 2012, 87(9-12), 272-290.

8. B. Honeyman, M. Bedzyk, M. Hersam, H. Jin, C.R. Kinser, Nanoscape, 2005, 2(1), 89-95.

9. A. Salomon, T. Böcking, J. J. Gooding, D. Cahen, Nano Letters, 2006, 6(12), $2873-$ 2876.

10. P. Fernández, "Materiales carbonosos de distinta índole para el almacenamiento y la conversión electroquímica de energía", tesis doctoral para optar el título de Doctor en Ciencias Químicas, Universidad Nacional de la Plata UNLP, 2011.

11. J. Kim. Probing Organic Self-Assembled Monolayers (SAMs) on Silicon by FTIR with Single Reflectance ATR, PIKE technologies.

12. A.B. Sieval, R. Opitz, H. Maas, M. Schoeman, G. Meijer, F. Vergeldt, et. al., Langmuir, 2000, 16(26), 10359-10368.

13. M. Li, "Modification of silicon by self-assembled monolayers for application in nanoelectronics and biology", tesis doctoral para optar el título de Doctor en Física y Astronomía, Universidad de Nueva Jersey, 2007.

14. A.B. Sieval, A.L. Demirel, J.W.M. Nissink, M.R. Linford, J.H. van der Maas, W.H. de Jeu et. al., Langmuir, 1998, 14(7), 1759-1768.

15. J.H. Song, M.J. Sailor, Inorganic Chemistry, 1998, 37(13), 3355-3360. 